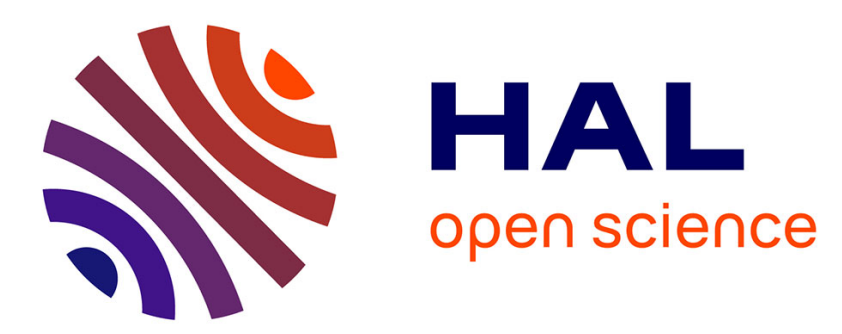

\title{
Observation of the asphericity of 4 f-electron density and its relation to the magnetic anisotropy axis in single-molecule magnets
}

Chen Gao, Alessandro Genoni, Song Gao, Shangda Jiang, Alessandro Soncini, Jacob Overgaard

\section{To cite this version:}

Chen Gao, Alessandro Genoni, Song Gao, Shangda Jiang, Alessandro Soncini, et al.. Observation of the asphericity of $4 \mathrm{f}$-electron density and its relation to the magnetic anisotropy axis in single-molecule magnets. Nature Chemistry, 2020, 12 (2), pp.213-219. 10.1038/s41557-019-0387-6 . hal-02488685

\section{HAL Id: hal-02488685 \\ https://hal.univ-lorraine.fr/hal-02488685}

Submitted on 28 May 2020

HAL is a multi-disciplinary open access archive for the deposit and dissemination of scientific research documents, whether they are published or not. The documents may come from teaching and research institutions in France or abroad, or from public or private research centers.
L'archive ouverte pluridisciplinaire HAL, est destinée au dépôt et à la diffusion de documents scientifiques de niveau recherche, publiés ou non, émanant des établissements d'enseignement et de recherche français ou étrangers, des laboratoires publics ou privés. 


\title{
Observation of the asphericity of $4 \mathrm{f}$-electron density and its relation to the magnetic anisotropy axis in single-molecule magnets
}

Chen Gao, ${ }^{a}$ Alessandro Genoni, ${ }^{b}$ Song Gao, ${ }^{c}$ Shangda Jiang, ${ }^{c^{*}}$ Alessandro Soncini, ${ }^{{ }^{*}}$ Jacob Overgaard $^{a} *$

${ }^{a}$ Department of Chemistry, Aarhus University, DK-8000 Aarhus C, Denmark

${ }^{\mathrm{b}}$ Université de Lorraine, CNRS, Laboratoire LPCT, 1 Boulevard Arago, F-57078, Metz, France

${ }^{c}$ Beijing National Laboratory of Molecular Science, State Key Laboratory of Rare Earth Materials Chemistry and Applications, College of Chemistry and Molecular Engineering, Peking University, Beijing 100871, P. R. China

${ }^{\mathrm{d}}$ School of Chemistry, University of Melbourne, 3010 Victoria, Australia

\begin{abstract}
The distribution of electrons in the $4 f$ orbitals of lanthanide ions is often assigned a crucial role in the design of single-molecule magnets (SMMs), which maintain a magnetization in zero external field. Optimal spatial complementarity between the $4 f$-electron density ( $4 f$-ED) and the ligand field is key to maximizing magnetic anisotropy — an important factor in the ability of lanthanide complexes to display SMM behaviour. Here, we have experimentally determined the ED distribution in two dysprosium molecular complexes by interpreting highresolution synchrotron X-ray diffraction with a multipole model. The ground-state $4 \mathrm{f}-\mathrm{ED}$ is indeed found to be an oblate ellipsoid — as is often deduced from a simplified Sievers model that assumes a pure $\mid \pm 15 / 2>$ ground-state doublet for the lanthanide ion. The large equatorial
\end{abstract}


asymmetry, determined via a model wavefunction, was found to contain significant $M_{J^{-}}$ mixing of $\mid \pm 11 / 2>$ and only $81 \%$ of $\mid \pm 15 / 2>$. The experimental molecular magnetic easy axes were recovered, and found to deviate by $13.1^{\circ}$ and $8.7^{\circ}$ from those obtained by ab initio calculations. 
Lanthanide ions continue to represent the focus of forefront multidisciplinary research, playing a crucial role in areas as diverse as magnetic resonance imaging ${ }^{1}$ and cell labelling, structure determination of large proteins via paramagnetic $\mathrm{NMR}^{2}$ and the development of building blocks for molecule-based storage devices, quantum computing ${ }^{3}$ and spintronics. ${ }^{4}$. Central to the development of all these applications is the non-uniform distribution of a few electrons amongst the lanthanides' seven $4 \mathrm{f}$ orbitals, and its consequences for the ensuing magnetic anisotropy. Magnetic anisotropy plays a particularly important role in the development of single-molecule magnets (SMMs), which possess the ability to maintain a magnetization in zero external field. Since the discovery of the SMM property, ${ }^{5-7}$ numerous molecular systems have been shown to exhibit restricted equilibration of magnetization ${ }^{8-14}$. The common origin of this behaviour is magnetic anisotropy, which expresses the differences in resulting magnetization as a function of the direction of the external magnetizing field relative to the molecular orientation.

Recently, Rinehart and Long ${ }^{15}$ presented an explanation for the relaxation of magnetization in lanthanide complexes. ${ }^{16}$ Their underlying idea was that the reversal of the magnetization from the state $M_{J}=-J$ to the state $M_{J}=+J$ via the intermediate $M_{J}$-states, i.e. neglecting quantum tunneling, could be interpreted as being accompanied by a $4 \mathrm{f}$ electron density (ED) redistribution. This rationalizes the energy barrier to magnetization reversal as being caused by a classical rotation of the $4 \mathrm{fED}$, tightly bounded to the magnetic moment orientation, into a configuration of energetically unfavorable $4 \mathrm{f}$-ligand electrostatic repulsion at the top of the barrier. Thus, the ease of this ED reorientation is strongly influenced by its electrostatic interactions with the surrounding ligand field. Consider, for instance, the trivalent dysprosium-ion, which has a $4 \mathrm{f}^{9}$ configuration and the ground state term is ${ }^{6} \mathrm{H}_{15 / 2}$. Basic theoretical calculations suggest that the valence ED for the $M_{J}=15 / 2$ state belonging to the ${ }^{6} \mathrm{H}_{15 / 2}$ multiplet has a pronounced oblate character - i.e. there is significantly less ED in the 
direction of the vertical Z-axis than in the equatorial XY-plane. ${ }^{17}$ If the surrounding ligand field complements this oblate ED shape by having most of its negative charge occupying the regions along the Z-axis, the electrostatic repulsion is minimized. Building upon these ideas, Chilton and Soncini created a program to calculate the energetically most favourable direction (or easy axis) of magnetization for Dy compounds with Dy exclusively in the $\mid M_{J}=$ $15 / 2>$ state, by minimizing the classical electrostatic repulsion energy between the charge distributions of the ligands and the Dy ion. ${ }^{18}$ It was soon realized that a linear ligand field would maximize the stabilization of the $\mid M_{J}= \pm 15 / 2>$, eventually leading to the discovery of the record-breaking dysprosocenium SMM. ${ }^{19-22}$

However, the above-described argumentation relies on a calculation of the pure $\mid M_{J}=15 / 2>$ state of an isolated atom, neglecting $M_{J}$-mixing effects, dynamical correlation effects, and covalent bonding effects. It is thus of importance to gain additional experimental insight into the properties of the ED to unambiguously prove this relationship, and the prime approach is to use high-resolution single-crystal X-ray diffraction. ${ }^{23}$ This method allows a complete experimental parameterization of the ED in the crystalline state, inherently including spinorbit effects and intermolecular interactions. These effects are exceedingly difficult to quantify by theoretical calculations, but are provided "for free" in the experiment. However, application of the method to heavy elements such as lanthanides remains an elusive target, primarily because of the lack of sufficiently accurate data to describe the spatially overlapping $4 \mathrm{f}$ and $5 \mathrm{p}$ electrons. This is evidenced by the fact that only two experimental ED studies of lanthanide compounds have been published, ${ }^{24,25}$ both of these having filled or empty $4 \mathrm{f}$ shells. This is not the case for Dy(III) with its partially filled $4 \mathrm{f}$ shell, and this study is therefore of significance for ED research. Key to the current success has been the combined use of synchrotron radiation, ${ }^{26-30}$ an experimental temperature of $20 \mathrm{~K},{ }^{31,32}$ and 
novel detector technology, thereby allowing high-resolution data with minimal systematic errors.

As benchmark compounds, we have chosen both known polymorphs of $\mathrm{Dy}(\mathrm{dbm})_{3}(\mathrm{bpy}),(\mathbf{1} \mathbf{A}$, Figure 1) and (1B), in which Dy(III) is coordinated to three molecules of dibenzoylmethanoate (dbm), and one 2,2'-bipyridine (bpy). Compound 1B is known to behave as an SMM with an energy barrier of $16.5 \mathrm{~K},{ }^{33}$ and some of us have just determined its magnetic susceptibility tensor using polarized neutron diffraction. ${ }^{34}$

\section{Results}

The main purpose of this study is to demonstrate the experimental determination of the ED distribution in lanthanide molecular complexes. Explicit details about how the total ED is obtained are given in the Methods section. In order to ensure the validity of this approach, we need to fulfil three conditions: Firstly, we probe the ED exclusively from the ground state.

The first excited state for $\mathbf{1 A}(\mathbf{1 B})$ is calculated to be $161 \mathrm{~cm}^{-1}\left(149 \mathrm{~cm}^{-1}\right)$ above the ground state for 1A (1B), and with an experimental temperature of $20 \mathrm{~K}$, their Boltzmann population is negligible. Secondly, while we do model the total ED including any covalency, any twocenter bonding involving the $4 \mathrm{f}$ electrons on the central Dy-ion and the surrounding ligand atoms is neglected during extraction of $4 \mathrm{f}$-orbital populations. It is well known that the $4 \mathrm{f}$ electrons in lanthanides are effectively shielded by the $5 \mathrm{~s}$ and $5 \mathrm{p}$ electrons, and the extent of electron sharing with the ligands involving the $4 \mathrm{f}$ electrons will be small. We have verified this assumption, as outlined in detail in the Supplementary Information (Section 3), by showing clear evidence that the chemical bonding involving Dy is primarily ionic. Thirdly, we verified the applicability of the multipole model to describe the ED in lanthanide systems by refining against noise-free theoretical structure factors. The development of a new procedure was required for the calculation of such structure factors based on a multi- 
configurational wavefunction. Using the simulated data, we obtain a highly reliable model, clearly confirming that the multipole approach has the capability to accurately describe the complex electronic distribution around Ln-ions.

\section{Experimental determination of the Dysprosium $4 \mathrm{f}$ orbital populations}

In this section, we thus assume that the Dy ${ }^{\mathrm{III}}$ ion is described by a purely atomic ED (see Methods, see Eq. (5)), whose departure from sphericity is solely determined by the nonuniform distribution of the nine electrons in the seven $4 f$ orbitals. This deviation results from the crystal field splitting of the ground spin-orbit multiplet (an assumption strongly supported by the found lack of covalency in the Dy-ligand bonding). Under this assumption, we have developed (Methods, Eq. (6)) a description of the first order reduced $4 f$-density matrix in terms of the experimentally measured multipoles which allows us to access the 4 -orbital populations (Table 1 and 2).

The ab initio calculations and magnetic data are consistent with a ground doublet dominated by $\mid M_{J}= \pm 15 / 2>$, if this is represented on a basis of $\left|J M_{J}\right\rangle$ multi-electron spin-orbit states, where the quantum number $M_{J}$ defines the projection of the total angular momentum $J$ along the magnetic anisotropy axis. Thus, using basis functions quantized along the magnetic easy axis, this leads to $4 \mathrm{f}$-orbital populations of 1.5 in each of the four $m_{l}= \pm 3, \pm 2$ functions and 1.0 in the remaining three $\left(m_{l}= \pm 1,0\right)$. The experimental $4 f$-orbitals populations defined along the ab initio quantization axis clearly show that the nine open-shell electrons are not uniformly distributed among the 4 -orbitals, but instead nearly consistent with the idealized occupation pattern predicted for a pure $\mid \pm 15 / 2>$ state. It is more meaningful to compare the electronic populations $N_{m}$ of different angular momentum shells, reported in columns A (experimental) and $C$ (theoretical). For a pure $\mid \pm 15 / 2>$ doublet, we expect the $m=3$ and $m=2$ 
shells to be populated with three electrons in each shell, the $m=1$ shell with two electrons, and the $m=0$ shell with one electron. For $\mathbf{1 A}$ it is clear that, aside from almost half an electron transferred between shells $m=2$ and $m=0$, the overall experimental populations indicate a multi-electron ground state wavefunction rather dominated by a $\pm 15 / 2>$ doublet. This picture is even clearer for $\mathbf{1 B}$, where again half an electron from $m=2$ relative to the pure $\mid \pm 15 / 2>$ doublet state is re-distributed, this time evenly among the rest of the orbitals. The experimental populations are thus also consistent with the Hund's rule character of such state, according to which the $4 f$ orbital shell with the highest angular momentum should be the most populated.

The experimental multipole model of the ED allows us to estimate all 28 unique matrix elements of the first order reduced $4 f$-density matrix. These show (Tables S5-6) that the experimental density matrix is not diagonal. We can rationalize this based on the following arguments: Firstly, the experimental magnetic anisotropy axes might not lie in the same direction as the ab initio axes, and rotation of the basis of tesseral harmonics (i.e. the real spherical multipoles, for details see SI, section 6, Eq. (S2)) from the experimental magnetic anisotropy axis to our chosen reference frame would introduce off-diagonal elements in the reduced density matrix. We show in the next section that the experimental easy axis forms an angle of only $13^{\circ}$ for $\mathbf{1 A}$ and $8.7^{\circ}$ for $\mathbf{1 B}$ with the ab initio easy axis. Secondly, even if the directions of the experimental and theoretical easy axes coincide, in the absence of high axial symmetry the hypothesis of a pure $\mid \pm 15 / 2>$ is an idealization for a molecule. The effect of going beyond the pure $\mid \pm 15 / 2>$ approximation is treated in the final section. Finally, despite our best efforts to use state-of-the-art experimental and theoretical methods, experimental uncertainties and theoretical approximations necessarily introduced will account for some of the discrepancies between experiment and theory. 


\section{Spatial shape of the 4 f electron cloud}

We now use the experimentally determined multipolar expansion to analyze the shape of the $4 f \mathrm{ED}$ in the two molecules, in the spirit of the approach by which Sievers illustrated the theoretical lanthanide densities (for details, see SI, Section 7). ${ }^{17}$ When Dy ${ }^{\mathrm{III}}$ is in a ground state strongly dominated by the $\mid \pm 15 / 2>$ component, we expect the magnetic easy-axis to lie along the axis of the associated oblate ellipsoid representing the $4 \mathrm{f}$ ED. We are thus left with the task of finding this unique direction defining the $4 \mathrm{f} \mathrm{ED.}$

To accomplish this, we characterize the oblate character of Eq. (6) by ignoring any harmonic of order higher than two, thus keeping in the expansion only the monopolar $\left(Z_{00}\right)$ and the five quadrupolar $\left(Z_{2 m}^{\lambda}\right)$ terms. We can write (see Section 6 in SI) the experimental 4 f density for 1A and 1B in the truncated quadrupolar approximation $\rho_{2}$ as (using the experimental multipole values, see Table S5):

$$
\begin{aligned}
& \rho_{2}^{\exp , 1 \mathrm{~A}}(\theta, \phi)=9.0 \tilde{Z}_{00}-0.26 \tilde{Z}_{20}-0.02 \tilde{Z}_{21}^{+}-0.08 \tilde{Z}_{21}^{-}+0.05 \tilde{Z}_{22}^{+}-0.07 \tilde{Z}_{22}^{-} \\
& \rho_{2}^{\exp , 1 \mathrm{~B}}(\theta, \phi)=9.0 \tilde{Z}_{00}-0.32 \tilde{Z}_{20}+0.03 \tilde{Z}_{21}^{+}+0.09 \tilde{Z}_{21}^{-}-0.07 \tilde{Z}_{22}^{+}+0.07 \tilde{Z}_{22}^{-}
\end{aligned}
$$

By rotating Eq. (1) in the ellipsoid principal axes frame, the ellipsoid surface represented by Eq. (1) may be simplified to its canonical form, Eq. (2), (for details see Section 7 in the SI):

$$
\rho_{2}(\theta, \phi)=A_{00} \tilde{Z}_{00}+A_{20} \tilde{Z}_{20}^{\prime}(\theta)+A_{22} \tilde{Z}_{22}^{\prime+}(\theta, \phi)
$$

Upon this rotation we may determine (i) its oblate character, as predicted by the ab initio calculations, and (ii) the direction of its shortest principal semi-axis corresponding to the direction of the magnetic easy axis. We obtain:

$$
\begin{aligned}
& \rho_{2}^{\exp , 1 \mathrm{~A}}(\theta, \phi)=9.0 \tilde{Z}_{00}-0.27 \tilde{Z}_{20}^{\prime}(\theta)+0.08 \tilde{Z}_{22}^{\prime+}(\theta, \phi) \\
& \rho_{2}^{\exp , 1 \mathrm{~B}}(\theta, \phi)=9.0 \tilde{Z}_{00}-0.33 \tilde{Z}_{20}^{\prime}(\theta)+0.10 \tilde{Z}_{22}^{\prime+}(\theta, \phi)
\end{aligned}
$$


This confirms that we have experimentally probed the strong oblate ellipsoidal character of the Dy ${ }^{\text {III }}$ ion, independently for both complexes investigated here. Eq. (3) reveal a sizeable axial quadrupolar coefficient for $\mathbf{1 A}\left(A_{20}=-0.273\right)$ and especially for $\mathbf{1 B}\left(A_{20}=-0.329\right)$, both close to the axial quadrupolar coefficient of the ideal oblate $\mid \pm 15 / 2>$ states, $A_{20}( \pm 15 / 2)=$ -0.321 (see SI, Section 6). It is noteworthy that while the pure states $\mid \pm 13 / 2>$ and $\mid \pm 11 / 2>$ also exhibit oblate charge distributions, they give rise to significantly smaller axial quadrupoles $\left(A_{20}( \pm 13 / 2)=-0.193, A_{20}( \pm 11 / 2)=-0.083\right)$. Again, this is strong indications that the experimental oblate shape of the $4 \mathrm{f} \mathrm{ED} \mathrm{measured} \mathrm{here} \mathrm{is} \mathrm{associated} \mathrm{to} \mathrm{a} \mathrm{ground} \mathrm{state}$ dominated by the $\mid \pm 15 / 2>$ doublet.

From the eigenvalues of the rotation matrix turning Eq. (1) into Eq. (3), we can quantify the angular density along the principal axes. For 1A $(\mathbf{1 B})$, we find $c=0.6034(c=0.5800)$ along the experimental easy-axis $Z$, where depletion of $4 \mathrm{f} E D$ occurs. These values are to be compared with the values derived from the pure $\mid \pm 15 / 2>$ state of $c=0.5836$ along the $Z$ easyaxis (see SI, Section 7). Since the $z$-axis of the experimental $x y z$-frame was deliberately chosen to coincide with the ab initio magnetic easy-axis, the angle between the direction of the experimentally determined short principal axis $c$, characterizing the spatial direction of maximal depletion of $4 \mathrm{f}-\mathrm{ED}$, and the ab initio easy axis, is the Euler angle $\beta=13.1^{\circ}$ for $\mathbf{1 A}$ and $\beta=8.7^{\circ}$ for $\mathbf{1 B}$. We illustrate in Figure 2 the relative orientation of experimental and ab initio magnetic axes, together with a plot of the ab initio (see Eq. (4)) and experimental (Eq. (1)) $4 \mathrm{f}$ EDs in the ellipsoidal approximation.

We thus find a very small difference between the measured axial quadrupolar coefficient $A_{20}$, and that predicted by theory for a Dy(III) ion in its maximally oblate state $\mid \pm 15 / 2>$. This leads to the conclusion that our measurements of the asphericity and the absolute spatial orientation 
of the $4 \mathrm{f} E D$ are in agreement with a ground doublet dominated by a $\mid \pm 15 / 2>$ state. However, we also note that if the ground state were described by an almost pure $\mid \pm 15 / 2>$ doublet, the rhombic quadrupolar coefficient $\mathrm{A}_{22}$ should be negligibly small, which is in stark contrast to what we find here. In the next section, we therefore discuss the origin of this XY-asymmetry. We remind that there is a comparatively large $\mathrm{XY}$-asymmetry in the atomic magnetic susceptibility for Dy in $\mathbf{1 B}$, as determined using polarized neutron diffraction. ${ }^{34}$ In that study, we obtain directly the individual components of the atomic susceptibility tensor, and find the ratio of the two XY susceptibilities to be 1.53 .

\section{XY-asymmetry and ground state composition}

As shown in Eq. (3), the rhombic contributions are sizeable $\left(A_{22+} \sim 0.1\right)$, showing that the $4 \mathrm{f}$ ED displays a larger accumulation along $X$ than along $Y$. Importantly, such rhombic departure is not allowed in Sievers' theory. ${ }^{17}$ To investigate this matter further we used the full ab initio wavefunctions of the ground doublet $\left|\Psi_{ \pm}\right\rangle=\sum_{M=-J}^{+J} a_{M}^{ \pm}|J M\rangle$ (see Table S4 for the list of $a_{M}^{ \pm}$), to compute the theoretical multipoles of the $4 \mathrm{f} \mathrm{ED} \mathrm{(see} \mathrm{Methods,} \mathrm{Eq.} \mathrm{(7),} \mathrm{and} \mathrm{SI,} \mathrm{Section} \mathrm{8).}$ We note that the ab initio calculations predict a ground doublet dominated by $\mid \pm 15 / 2>$ ( $92 \%)$, as well as a non-negligible contribution from $\mid \pm 11 / 2>(\sim 8 \%)$.

Applying the developed Eq. (7), with the results of our ab initio calculations, we get the following expression for the theoretical density in the quadrupole approximation:

$$
\rho_{2}^{\text {theo, } 1 \mathrm{~A}}(\theta, \phi)=9.0 \tilde{Z}_{00}-0.30 \tilde{Z}_{20}^{\prime}(\theta)+0.03 \tilde{Z}_{22}^{\prime+}(\theta, \phi)
$$

We can see from Eq. (4) that the corrections to simple Sievers' theory brought about by the mix in the ground doublet of a $\mid \pm 11 / 2>$ component are quite sizeable, and exactly in the direction predicted by our experimental data, with a non-negligible rhombic term accounting for the mixing. However, the $8 \%$ mixing of $\mid \pm 11 / 2>$ obtained via the popular ab initio method 
used here only accounts for roughly about a third of the measured rhombic distortion of the ED. This is yet another indication of the tendency of these programs to predict ground states with too high purity. For instance, this bias is highlighted by comparison of simulated and measured magnetic susceptibility data of $\mathbf{1 A}$, as shown below in Figure 3. The limiting value of $\chi \mathrm{T}$ of a Kramers doublet for $\mathrm{T}$ approaching zero is given by $(\chi T)_{T=0}=\frac{N \mu_{B}^{2}}{3 k_{B}} \times \frac{g_{x}^{2}+g_{y}^{2}+g_{z}^{2}}{4}$, which equals $11.8 \mathrm{emu} \mathrm{K} \mathrm{mol}^{-1}$ for the ab initio result, while experimentally it is around 10.5 emu $\mathrm{K} \mathrm{mol}^{-1}$. If instead we use the g-values obtained from the highly mixed ground state ${ }^{35}$ resulting from the fit to the experimental $\mathrm{ED}$, we obtain a value of $11.1 \mathrm{emu} \mathrm{K} \mathrm{mol}{ }^{-1}$, in much better correspondence with the experimental magnetization measurement.

To examine in more detail the degree of mixing that accounts for the experimental rhombicity, we consider first a simple two-level model of the ground Kramer's doublet consisting of $| \pm 11 / 2\rangle$ and $| \pm 15 / 2\rangle$, which allows us to parameterize the canonical quadrupoles in terms of a mixing angle $\alpha$ (see also SI, Section 8). If we maximize $\mathrm{A}_{22}$ under the constraint that $\left|\mathrm{A}_{20}\right|$ remains in the range $0.25 \sim 0.3$, we find that this simple two-level model allows for at most $32 \%$ mixing of $\mid \pm 11 / 2>\left(\alpha=68.9^{\circ}\right)$, leading to $A_{20}=-0.25, \mathrm{~A}_{22}=$ 0.048 , which is, however, still only about $60 \%$ of the experimental rhombic mixing obtained from the ED for $\mathbf{1 A}$.

A more flexible model allows mixing of those eight $\left|\mathrm{M}_{\mathbf{J}}\right\rangle$ states that differ by 2 units of angular momentum from each other, starting from $\mid+15 / 2>$ and is parameterized by the 7 mixing angles $\alpha_{1}, \alpha_{2}, \ldots, \alpha_{7}$ as:

$$
\begin{aligned}
|\mathrm{KD}+\rangle= & \cos \left(\frac{\alpha_{1}}{2}\right)\left|\frac{15}{2}\right\rangle+\sin \left(\frac{\alpha_{1}}{2}\right) \cos \left(\frac{\alpha_{2}}{2}\right)\left|\frac{11}{2}\right\rangle+\sin \left(\frac{\alpha_{1}}{2}\right) \sin \left(\frac{\alpha_{2}}{2}\right) \cos \left(\frac{\alpha_{3}}{2}\right)\left|\frac{7}{2}\right\rangle+\cdots \\
& \left.+\sin \left(\frac{\alpha_{1}}{2}\right) \sin \left(\frac{\alpha_{2}}{2}\right) \cdots \cos \left(\frac{\alpha_{7}}{2}\right) \mid-\frac{9}{2}\right)+\sin \left(\frac{\alpha_{1}}{2}\right) \sin \left(\frac{\alpha_{2}}{2}\right) \cdots \sin \left(\frac{\alpha_{7}}{2}\right)\left|-\frac{13}{2}\right\rangle
\end{aligned}
$$


This model wavefunction Eq. (4a) is expected to lead to the maximal rhombic quadrupole term in the associated ED represented in a canonical form, consistent with our Eqs. (7) and (S13). We use $\alpha_{1}$ to constrain the axial quadrupole so that $0.3 \geq\left|\mathrm{A}_{20}\right| \geq 0.25$, consistent with experiment within an expected error of 0.03 on the multipole parameters, while the remaining six angles are varied to maximize the rhombic quadrupole coefficient $\left|\mathrm{A}_{22}\right|$. We find that the best match to the experimental canonical quadrupoles consist of $\mathrm{A}_{20}=-0.25, \mathrm{~A}_{22}=0.07$ (closely resembling the experimental values for $\mathbf{1 A}, \mathrm{A}_{20}=-0.27, \mathrm{~A}_{22}=0.08$ ), obtained using the substantially mixed wavefunction with $\mathbf{8 1 \%}|\mathbf{1 5} / 2>, 5.6 \%| 11 / 2>, 3.8 \%|7 / 2>, 4 \%| 3 / 2>$, $3.4 \%|-1 / 2>, 1.7 \%|-5 / 2>, 0.4 \%|-9 / 2>, 0.04 \%|-13 / 2>$.

Using all 28 ab initio multipoles calculated via Eq. (7), we obtain a new set of $4 \mathrm{f}$ populations defined in the ellipsoidal principal axes (columns E in Table $1 \& 2$ ). These vary little with respect to the populations of the pure $\mid \pm 15 / 2>$ states, but we note that the changes for the $m=0, m=1$ and $m=2$ shells occur in the direction observed in the experiment, i.e. increasing the populations of the $m=0$ and $m=1$ and reducing the $m=2$ shells. However, at variance with the experiment, we also observe a slight decrease in the population of the $m=3$ shell.

\section{Discussion}

In conclusion, we have used high-resolution, low-temperature synchrotron X-ray diffraction data collected for two independent polymorphs of dysprosium-based SMMs to obtain experimental multipole models for the total ED distribution. Using these, we have extracted 4f orbital populations and found them in quite good agreement with those resulting from a ground doublet dominated by $\mid M_{J}= \pm 15 / 2>$. Ab initio results rationalize the appearance of a rhombic term in the quadrupolar density in terms of a sizeable contribution from the $\mid M_{J}=$ $\pm 11 / 2>$ state, which still preserves a $92 \%$ dominance of $\mid M_{J}= \pm 15 / 2>$. However, a more quantitative agreement with the observed stronger rhombic XY-asymmetry could only be 
recovered via a model wavefunction allowing a stronger mixing, leading to a ground $\mathrm{KD}$ dominated only by $81 \%$ by of $\mid M_{J}= \pm 15 / 2>$, in a clear confirmation of the failure of current ab initio models to predict substantial ground state mixing.

By analysis of the spatial shape of the $4 \mathrm{f} E D$ in the two compounds we have unambiguously proven that the $4 \mathrm{f}-\mathrm{ED}$ associated with the $\mathrm{Dy}^{3+}$-ion is oblate, however less cylindrically symmetrical than expected. In addition, we have derived the magnetic easy axis direction from the rotation of the quadrupoles. This constitutes an experimentally validated link between electronic structure and magnetic properties. Importantly, this study provides experimental observations of the $4 \mathrm{f} \mathrm{ED}$ in functional single-ion magnets, and expands upon the simple yet effective electrostatic model, by giving a much more detailed view of the valence density shape. We note that the present magnetic anisotropy axis of $\mathbf{1 B}$ is in full agreement with that found using polarized neutron diffraction on the same compound, as well as aligned with both ab-initio and the electrostatic model results.

\section{Methods}

\section{Synthesis}

Compound 1 was synthesized according to literature instructions. ${ }^{33}$ Crystals were obtained by recrystallization from evaporation of 1:1 ethanol/acetone solution.

\section{Single crystal $\mathrm{X}$-ray diffraction data collection and reduction}

Single crystal X-ray diffraction data was collected at beamline BL02B1 at SPring8 in Japan. A single crystal of 1A with dimensions $110 \times 80 \times 55 \mu \mathrm{m}$ and of $\mathbf{1 B}$ of $60 \times 80 \times 90 \mu \mathrm{m}$ was mounted directly on a pointed glass tip using epoxy glue and cooled to $20 \mathrm{~K}$ using a He-flow. The diffraction data was measured using Fuji Imaging Plate detector technology. The data was collected using $3^{\circ} \omega$-scans, and a total of 59 frames for $\mathbf{1 A}$ and 116 frames for $\mathbf{1 B}$ 
provided datasets with completeness and redundancy of $96.7 \%$ and 2.7 for $1 \mathrm{~A}$ and $97.9 \%$ and 5.5 for 1B. The resolution was $0.43 \AA$ for $\mathbf{1 A}$ and $0.35 \AA$ for $\mathbf{1 B}$. The data was integrated using a box-sum method in which we reject all partial reflections. The integrated reflections were scaled using the software RAPID_AUTO, and the program SORTAV ${ }^{33,36}$ was used to discard outlier reflections and merge equivalent reflections, providing internal agreement factors of $2.3 \%(\mathbf{1 A})$ and $5.4 \%(\mathbf{1 B})$

\section{Experimental multipole modeling.}

The Hansen-Coppens model: ${ }^{30}$

$$
\rho_{\mathrm{at}}(\mathbf{r})=P_{c} \rho_{\text {core }}(\mathbf{r})+P_{v} \kappa^{\prime 3} \rho_{\text {valence }}\left(\kappa^{\prime} r\right)+\sum_{l=\mathbf{0}}^{l_{\text {max }}} \kappa^{\prime \prime 3} R_{l}\left(\kappa^{\prime \prime} r\right) \sum_{\boldsymbol{m}=\mathbf{0}}^{l} P_{l m \pm} d_{l m \pm}(\theta, \phi) .
$$

with $1_{\max }=6$ was used to describe the charge density. Radial functions are from bank files used by XD2006. The effectiveness of the model is tested with the refinement on theoretical structure factors. The crystal structure was solved using SHELXT, ${ }^{37}$ and refined using SHELXL ${ }^{38}$ in Olex $2,{ }^{39}$ before it was imported into $\mathrm{XD}^{40}$ for initial multipole modeling. The final refinements were carried out in JANA2006 ${ }^{41}$. More details can be found in SI.

\section{Computational details}

The electronic structure calculations of $\mathbf{1}$ was done with CASSCF/RASSI/SINGLE_ANISO implemented in the MOLCAS 8.2 package $^{42}$. Complete active-space self-consistent field (CASSCF) calculation is carried out with on the X-ray structure. For all atoms, basis sets are atomic natural orbitals from MOLCAS ANO-RCC library. ANO-RCC-VTZP for the Dy ion, VTZ for close $\mathrm{N}$ and $\mathrm{O}, \mathrm{VDZ}$ for the other atoms. The calculations employed the second order Douglas-Kroll-Hess Hamiltonian, where scalar relativistic contractions were taken into account in the basis set and the spin-orbit coupling was handled separately in the restricted 
active space state interaction (RASSI-SO) procedure. The active electrons in seven active spaces include all the $9 \mathrm{f}$ electrons in the CASSCF calculation. We calculated all the roots in the active space. We have mixed the maximum number of spin-free states, which was possible with our hardware (all from 21 sextets, 128 from 224 quadruplets and 130 from 490 doublets). The magnetic properties were obtained using SINGLE_ANISO. ${ }^{43}$ Energy level diagram, g-tensors of the ground and first excited states, and projections of wavefunctions in the effective 15/2 pseudo spin space are reported in the Supplementary Information (Section $5)$.

Theoretical structure factors were calculated analytically up to a resolution limit of $\sin (\theta) / \lambda=$ $1.7 \AA^{-1}$ by exploiting spin orbit natural orbitals (SONO), whose orbital coefficients file have been obtained through the RASSI program in MOLCAS $8.2^{42}$, and by placing molecule $\mathbf{1}$ in a large $25 \AA \times 25 \AA \times 25 \AA$ cubic cell. The calculations were performed using a routine of the XC-ELMO program ${ }^{44}$ that implements an extension of the Obara-Saika and Head-GordonPople recurrence relations for molecular integrals ${ }^{45,46}$ to the computations of the Fourier transforms of basis functions products. More details can be found in SI, Section 10. Computation of theoretical structure factors.

\section{Theoretical analysis of the multipolar expansion of the $4 \mathrm{f}$ ED}

As detailed in the Supplementary Information (Section 6), the atomic 4f ED is

$$
\rho_{4 f}(\mathbf{r})=|\varphi(r)|^{2} \sum_{m_{1} \lambda_{1}, m_{2} \lambda_{2}} D_{m_{1} \lambda_{1}, m_{2} \lambda_{2}} Z_{3 m_{1}}^{\lambda_{1}}(\theta, \phi) Z_{3 m_{2}}^{\lambda_{2}}(\theta, \phi)
$$

where $D_{m_{1} \lambda_{1}, m_{2} \lambda_{2}}$ is the $7 \times 7$ reduced density matrix represented on a basis of real $4 \mathrm{f}$ atomic orbitals $\psi_{4 f, m \lambda}(r, \theta, \phi)=\varphi(r) Z_{3 m}^{\lambda}(\theta, \phi)$, featuring 28 unique elements, including the seven 
4f orbital populations (diagonal elements). Eq. (5) can be exactly re-expressed as a finite linear combination of tesseral harmonics $Z_{3 m}^{\lambda}(\theta, \phi)$ (see SI for details):

$$
\rho_{4 f}(\mathbf{r})=|\varphi(r)|^{2} \sum_{k}^{0,2,4,6} \sum_{q \lambda} \mu_{k q \lambda} \Omega_{k q \lambda} Z_{k q}^{\lambda}(\theta, \phi)
$$

where the 28 coefficients $\mu_{k q \lambda}$ are the $4 \mathrm{f}$ multipoles measured in this work. Knowledge of the 28 multipoles in Eq. (6) gives access to all 28 elements of the reduced density matrix $D_{m_{1} \lambda_{1}, m_{2} \lambda_{2}}$ (and vice versa) by solving the linear system Eq. (S4), a strategy that was used here to determine the $4 \mathrm{f}$ orbital experimental populations reported in Table 1.

To determine theoretical multipole coefficients $\mu_{k q \lambda}$ in Eq. (6), using our ab initio results, we generalized the Sievers $\operatorname{model}^{17}$ valid for a pure M-component of a spin-orbit multiplet, to a crystal field level that is an arbitrary mix of M-levels, as detailed the SI (Section 8). Given the ab initio ground Kramers doublet wavefunction $|\Psi\rangle=\sum_{M=-J}^{+J} a_{M}|J M\rangle$ for the $\mathrm{Dy}^{3+}$ complex (reported in Table S4), we thus obtain the theoretical multipoles as (see also Eq. $(\mathrm{S} 13))$ :

$$
\begin{gathered}
\mu_{k q \lambda}=N_{q} e^{-i \pi 2^{\lambda}}\left\langle L S J\left\|O^{k}\right\| L S J\right\rangle \sum_{M_{1} \geq M_{2}=-J}^{+J}(-1)^{J-M_{1}+q}\left(a_{M_{1}}^{*} a_{M_{2}}\right. \\
\left.+\lambda a_{M_{2}}^{*} a_{M_{1}}\right)\left(\begin{array}{ccc}
J & k & J \\
-M_{1} & q & M_{2}
\end{array}\right)
\end{gathered}
$$

where $\left\langle L S J \| O^{k}|| L S J\right\rangle$ is the same reduced matrix element derived by Sievers ${ }^{17}$, and $N_{\mathrm{q}}=1 / 2$ $\left(N_{\mathrm{q}}=1 / \sqrt{ } 2\right)$ if $q=0(q>0)$. Via Eq. (7) we computed the ab initio multipoles (Table S5), hence via Eq. (S4) the ab initio $4 \mathrm{f}$ orbital populations (Tables 1 and 2), and density matrix (Table S6).

\section{ASSOCIATED CONTENT}


Details about the diffraction data collection, reduction and analysis. Computational details and results of energy levels, spatial shape of the ED, ellipsoid fitting of surfaces. (PDF)

\section{AUTHOR INFORMATION}

Corresponding Authors:

*jacobo@chem.au.dk (J.O.)

*asoncini@unimelb.edu.au (A.S.)

*jiangsd@pku.edu.cn (S.J.)

\section{Author Contributions}

J.O. designed the study, C.G. analysed experimental data, A.S. performed the theoretical analysis, S.J. synthesized the crystals, A.G. calculated theoretical structure factors. J.O., A.S., C.G. co-wrote the manuscript, with the help of the other authors.

Competing Interests

The authors declare no competing interests.

\section{ACKNOWLEDGMENT}

The authors are deeply grateful to Dr Vaclav Petricek for discussions about multipole modelling of lanthanide compounds using Jana2006. Mattia Sist, Venkatesha Hathwar, Hidetaka Kasai and Kunihisa Sugimoto are thanked for their help during synchrotron data collection. A.S. acknowledges support from the Australian Research Council, Future Fellowship No. FT1800100519. JO acknowledges the financial support from Independent Research Foundation Denmark, the Danish National Research Foundation (DNRF-93), VILLUM FOUNDATION and Danscatt. The synchrotron experiment was performed on 
beamline BL02B2 at SPring-8 with the approval of the Japan Synchrotron Radiation

Research Institute (JASRI) as a Partner User (Proposal No. 2016B0078).

Data availability.

Crystallographic data for the structures reported in this Article have been deposited at the Cambridge Crystallographic Data Centre, under deposition numbers CCDC 1900925 (1A), 1900926 (1B). Copies of the data can be obtained free of charge via https://www.ccdc.cam.ac.uk/structures/. All other data supporting the findings of this study are available within the Article and its Supplementary Information, or from the corresponding author upon reasonable request.

\section{REFERENCES}

1 Bottrill, M., Kwok, L. \& Long, N. J. Lanthanides in magnetic resonance imaging. Chem. Soc. Rev. 35, 557-571, doi:10.1039/B516376P (2006).

2 Otting, G. Protein NMR Using Paramagnetic Ions. Annual Review of Biophysics 39, 387-405, doi:10.1146/annurev.biophys.093008.131321 (2010).

3 Leuenberger, M. N. \& Loss, D. Quantum computing in molecular magnets. Nature 410, 789-793, doi:10.1038/35071024 (2001).

4 Troiani, F. et al. Molecular engineering of antiferromagnetic rings for quantum computation. Physical Review Letters 94, doi:10.1103/PhysRevLett.94.207208 (2005).

5 Sanvito, S. Molecular spintronics. Chem. Soc. Rev. 40, 3336-3355, doi:10.1039/C1CS15047B (2011).

6 Lis, T. Preparation, structure, and magnetic properties of a dodecanuclear mixedvalence manganese carboxylate. Acta Crystallogr. Sect. B 36, 2042-2046, doi:10.1107/S0567740880007893 (1980).

7 Sessoli, R., Gatteschi, D., Caneschi, A. \& Novak, M. A. Magnetic Bistability in a Metal-Ion Cluster. Nature 365, 141-143, doi:10.1038/365141a0 (1993).

8 Sessoli, R. \& Powell, A. K. Strategies towards single molecule magnets based on lanthanide ions. Coord. Chem. Rev. 253, 2328-2341, doi:10.1016/j.ccr.2008.12.014 (2009).

9 Blagg, R. J., Muryn, C. A., McInnes, E. J. L., Tuna, F. \& Winpenny, R. E. P. Single Pyramid Magnets: Dy-5 Pyramids with Slow Magnetic Relaxation to $40 \mathrm{~K}$. Angew. Chem. Int. Ed. 50, 6530-6533, doi:10.1002/anie.201101932 (2011). 
10 Samuel, P. P. et al. Electronic Structure and Slow Magnetic Relaxation of LowCoordinate Cyclic Alkyl(amino) Carbene Stabilized Iron(I) Complexes. J. Am. Chem. Soc. 136, 11964-11971, doi:10.1021/ja5043116 (2014).

11 Mallah, T. et al. Magnetic Anisotropy in Pentacoordinate Ni(II) and Co(II) Complexes: Unraveling Electronic and Geometrical Contributions. Chem. Eur. J. 23, 3648-3657, doi:10.1002/chem.201604872 (2017).

12 Rinehart, J. D., Fang, M., Evans, W. J. \& Long, J. R. A N23- Radical-Bridged Terbium Complex Exhibiting Magnetic Hysteresis at 14 K. J. Am. Chem. Soc. 133, 14236-14239, doi:10.1021/ja206286h (2011).

13 Craig, G. A. \& Murrie, M. 3d single-ion magnets. Chem. Soc. Rev. 44, 2135-2147, doi:10.1039/C4CS00439F (2015).

14 Bar, A. K., Pichon, C. \& Sutter, J.-P. Magnetic anisotropy in two- to eightcoordinated transition-metal complexes: Recent developments in molecular magnetism. Coord. Chem. Rev. 308, 346-380, doi:10.1016/j.ccr.2015.06.013 (2016).

15 Rinehart, J. D. \& Long, J. R. Exploiting single-ion anisotropy in the design of felement single-molecule magnets. Chem. Sci. 2, 2078-2085, doi:10.1039/C1SC00513H (2011).

16 Molecular nanomagnets. (Oxford University Press, 2006).

17 Sievers, J. Asphericity of 4f-Shells in Their Hund Rule Ground-States. Z Phys B Con Mat 45, 289-296, doi:10.1007/Bf01321865 (1982).

18 Chilton, N. F., Collison, D., McInnes, E. J., Winpenny, R. E. \& Soncini, A. An electrostatic model for the determination of magnetic anisotropy in dysprosium complexes. Nat. Commun. 4, 2551, doi:10.1038/ncomms3551 (2013).

19 Goodwin, C. A. P., Ortu, F., Reta, D., Chilton, N. F. \& Mills, D. P. Molecular magnetic hysteresis at 60 kelvin in dysprosocenium. Nature 548, 439-442, doi:10.1038/nature23447 (2017).

20 Chilton, N. F., Goodwin, C. A. P., Mills, D. P. \& Winpenny, R. E. P. The first nearlinear bis(amide) f-block complex: a blueprint for a high temperature single molecule magnet. Chem. Commun. 51, 101-103, doi:10.1039/C4CC08312A (2015).

21 Gupta, S. K., Rajeshkumar, T., Rajaraman, G. \& Murugavel, R. An air-stable Dy(iii) single-ion magnet with high anisotropy barrier and blocking temperature. Chem. Sci. 7, 5181-5191, doi:10.1039/C6SC00279J (2016).

22 Meng, Y.-S. et al. Low-Coordinate Single-Ion Magnets by Intercalation of Lanthanides into a Phenol Matrix. Angew. Chem. Int. Ed. 57, 4673-4676, doi:10.1002/anie.201801223 (2018).

23 Koritsanszky, T. S. \& Coppens, P. Chemical applications of X-ray charge-density analysis. Chem Rev 101, 1583-1627, doi:10.1021/cr990112c (2001).

24 Ananyev, I. V., Nelyubina, Y. V., Puntus, L. N., Lyssenko, K. A. \& Eremenko, I. L. Peculiarities of metal-ligand bonding in europium trinitrate complexes: a viewpoint of comparative charge density analysis in crystals. Russian Chemical Bulletin 65, 1178-1188, doi:10.1007/s11172-016-1434-8 (2016).

25 Puntus, L. N., Lyssenko, K. A., Antipin, M. Y. \& Bünzli, J.-C. G. Role of Inner- and Outer-Sphere Bonding in the Sensitization of EuIII-Luminescence Deciphered by Combined Analysis of Experimental Electron Density Distribution Function and Photophysical Data. Inorg. Chem. 47, 11095-11107, doi:10.1021/ic801402u (2008).

26 Meindl, K., Herbst-Irmer, R. \& Henn, J. On the effect of neglecting anharmonic nuclear motion in charge density studies. Acta Crystallogr. Sect. A 66, 362-371, doi:10.1107/s0108767310006343 (2010).

27 Schmokel, M. S. et al. Comparative study of X-ray charge-density data on CoSb3. Acta Crystallogr. Sect. A 69, 570-582, doi:10.1107/s0108767313024458 (2013). 
28 Schmokel, M. S. et al. Testing the Concept of Hypervalency: Charge Density Analysis of K2SO4. Inorg. Chem. 51, 8607-8616, doi:10.1021/ic301372m (2012).

29 Clausen, H. F. et al. Intermolecular Interactions and Electrostatic Properties of the beta-Hydroquinone Apohost: Implications for Supramolecular Chemistry. J. Phys. Chem. A 115, 12962-12972, doi:10.1021/jp2041789 (2011).

30 Coppens, P., Iversen, B. \& Larsen, F. K. The use of synchrotron radiation in X-ray charge density analysis of coordination complexes. Coord. Chem. Rev. 249, 179-195 (2005).

31 Prokeš, K., Gukasov, A., Javorský, P., Sechovský, V. \& Mihalik, M. Magnetic anisotropy in UNiGa determined by polarized neutrons. Physica B: Condensed Matter 301, 255-260 (2001).

32 Iversen, B. B. et al. Accurate charge densities in days - use of synchrotrons, image plates and very low temperatures. Acta Crystallogr. Sect. B 55, 363-374, doi:10.1107/S0108768198010398 (1999).

33 Dong, Y., Yan, P., Zou, X. \& Li, G. Azacyclo-auxiliary ligand-tuned SMMs of dibenzoylmethane Dy(iii) complexes. Inorganic Chemistry Frontiers 2, 827-836, doi:10.1039/C5QI00079C (2015).

34 Klahn, E. A. et al. Mapping the Magnetic Anisotropy at the Atomic Scale in Dysprosium Single-Molecule Magnets. Chem. Eur. J. 24, 16576-16581, doi:10.1002/chem.201803300 (2018).

35 Vancoillie, S., Malmqvist, P.-Å. \& Pierloot, K. Calculation of EPR g Tensors for Transition-Metal Complexes Based on Multiconfigurational Perturbation Theory (CASPT2). ChemPhysChem 8, 1803-1815, doi:10.1002/cphc.200700128 (2007).

36 Blessing, R. H. Data Reduction and Error Analysis for Accurate Single Crystal Diffraction Intensities. Crystallogr. Rev. 1, 3-58, doi:10.1080/08893118708081678 (1987).

37 Blessing, R. H. \& Langs, D. A. Data averaging with normal down-weighting of outliers. J. Appl. Crystallogr. 20, 427-428, doi:10.1107/S0021889887086357 (1987).

38 Sheldrick, G. SHELXT - Integrated space-group and crystal-structure determination. Acta Crystallogr A 71, 3-8, doi:doi:10.1107/S2053273314026370 (2015).

39 Dolomanov, O. V., Bourhis, L. J., Gildea, R. J., Howard, J. A. K. \& Puschmann, H. OLEX2: a complete structure solution, refinement and analysis program. J. Appl. Crystallogr. 42, 339-341, doi:10.1107/s0021889808042726 (2009).

40 XD2006 - A Computer Program Package for Multipole Refinement, Topological Analysis of Charge Densities and Evaluation of Intermolecular Energies from Experimental and Theoretical Structure Factors (2006).

41 JANA2006: a crystallographic computing system (Institute of Physics Academy of Science, Prague, 2006).

42 Aquilante, F. et al. Molcas 8: New capabilities for multiconfigurational quantum chemical calculations across the periodic table. J Comput Chem 37, 506-541, doi:10.1002/jcc.24221 (2016).

43 Chibotaru, L. F. \& Ungur, L. Ab initio calculation of anisotropic magnetic properties of complexes. I. Unique definition of pseudospin Hamiltonians and their derivation. $J$. Chem. Phys. 137, 064112, doi:10.1063/1.4739763 (2012).

44 Genoni, A. X-ray Constrained Extremely Localized Molecular Orbitals: Theory and Critical Assessment of the New Technique. J. Chem. Theory Comput. 9, 3004-3019, doi:10.1021/ct400293m (2013).

45 Obara, S. \& Saika, A. Efficient recursive computation of molecular integrals over Cartesian Gaussian functions. J. Chem. Phys. 84, 3963-3974, doi:10.1063/1.450106 (1986). 
46 Head Gordon, M. \& Pople, J. A. A method for two electron Gaussian integral and integral derivative evaluation using recurrence relations. J. Chem. Phys. 89, 57775786, doi:10.1063/1.455553 (1988). 
Figure 1. ORTEP depiction of the solid-state molecular structure of 1A, with $50 \%$ probability ellipsoids. The eight atoms of the first coordination sphere are labeled along with the central Dy(1). Hydrogen atoms have been omitted for clarity.

Figure 2. Electron density isosurfaces and unique magnetic axes. a-d, Overlay plots of the molecular structures showing experimental (red) and ab initio (green) $4 \mathrm{f}$ ED and experimental ellipsoid principal axes represented by red and blue arrows. Red arrows indicate the shortest axis, "oblate direction" while the two blue arrows indicate the two longer axes. Also shown are the ab initio magnetic axes of the ground doublet, with green arrows showing the easy-axis and black arrows showing the perpendicular magnetic axes with quasi-zero gvalues. The isosurfaces have been calculated including all 28 multipoles for $\mathbf{1 A}$ (a) and $\mathbf{1 B}$ (c), and in the ellipsoidal (monopole+quadrupoles) approximation for $\mathbf{1 A}$ (b) and 1B (d), as described by Eq. (1) (experimental) and Eq. (4) (ab initio). Colour code: grey, carbon; white, hydrogen; blue, nitrogen; red, oxygen.

Figure 3. Temperature dependence of the magnetic susceptibility $(\chi \mathrm{T})$ for 1A. The experimental values are shown with the dotted line, while the calculated values are shown using the solid line. Polymorph $\mathbf{1 A}$ is the preferred product, and we have therefore not been able to obtain sufficient amounts of $\mathbf{1 B}$ to enable magnetic measurement of this polymorph.

Table 1. 4f-orbital populations for 1A. The experimental values are based on a reference frame with the z-axis along the ab initio easy axis. Columns show: A (m-shell populations from experiment); B (m-shell populations from experiment, using easy axis as z-axis); C (mshell populations for pure $\mid \pm 15 / 2>$ ); D (m-shell populations from ab initio).

\begin{tabular}{clllll}
\hline Orbital & $m_{l}$ & $A$ & $B$ & $C$ & $D$ \\
\hline $4 f_{z^{3}}$ & 0 & 1.40 & 1.25 & 1.00 & 1.02 \\
$4 f_{x z^{2}}$ & \pm 1 & 2.10 & 2.23 & 2.00 & 2.05 \\
$4 f_{y z^{2}}$ & & & & & \\
$4 f_{z\left(x^{2}-y^{2}\right)}$ & \pm 2 & 2.33 & 2.33 & 3.00 & 2.94 \\
$4 f_{x y z}$ & & 3.19 & 3.00 & 2.99 \\
$4 f_{x\left(x^{2}-3 y^{2}\right)} \pm 3$ & 3.17 & & &
\end{tabular}


$4 f_{y\left(3 x^{2}-y^{2}\right)}$

Table 2. 4 -orbital populations for 1B. The experimental values are based on a reference frame with the z-axis along the ab initio easy axis. Columns show: A (m-shell populations from experiment); B (m-shell populations from experiment, using easy axis as $\mathrm{z}$-axis); $\mathrm{C}$ (mshell populations for pure $\mid \pm 15 / 2>$ ); D (m-shell populations from ab initio).

\begin{tabular}{clllll}
\hline Orbital & $m_{l}$ & $A$ & $B$ & $C$ & $D$ \\
\hline $4 f_{z^{3}}$ & 0 & 1.16 & 1.11 & 1.00 & 1.02 \\
$4 f_{x z^{2}}$ & \pm 1 & 2.13 & 2.25 & 2.00 & 2.06 \\
$4 f_{y z^{2}}$ & & & & & \\
$4 f_{z\left(x^{2}-y^{2}\right)}$ & \pm 2 & 2.51 & 2.37 & 3.00 & 2.93 \\
$4 f_{x y z}$ & & & & & \\
$4 f_{x\left(x^{2}-3 y^{2}\right)}$ & \pm 3 & 3.20 & 3.27 & 3.00 & 2.98 \\
$4 f_{y\left(3 x^{2}-y^{2}\right)}$ & & & & &
\end{tabular}



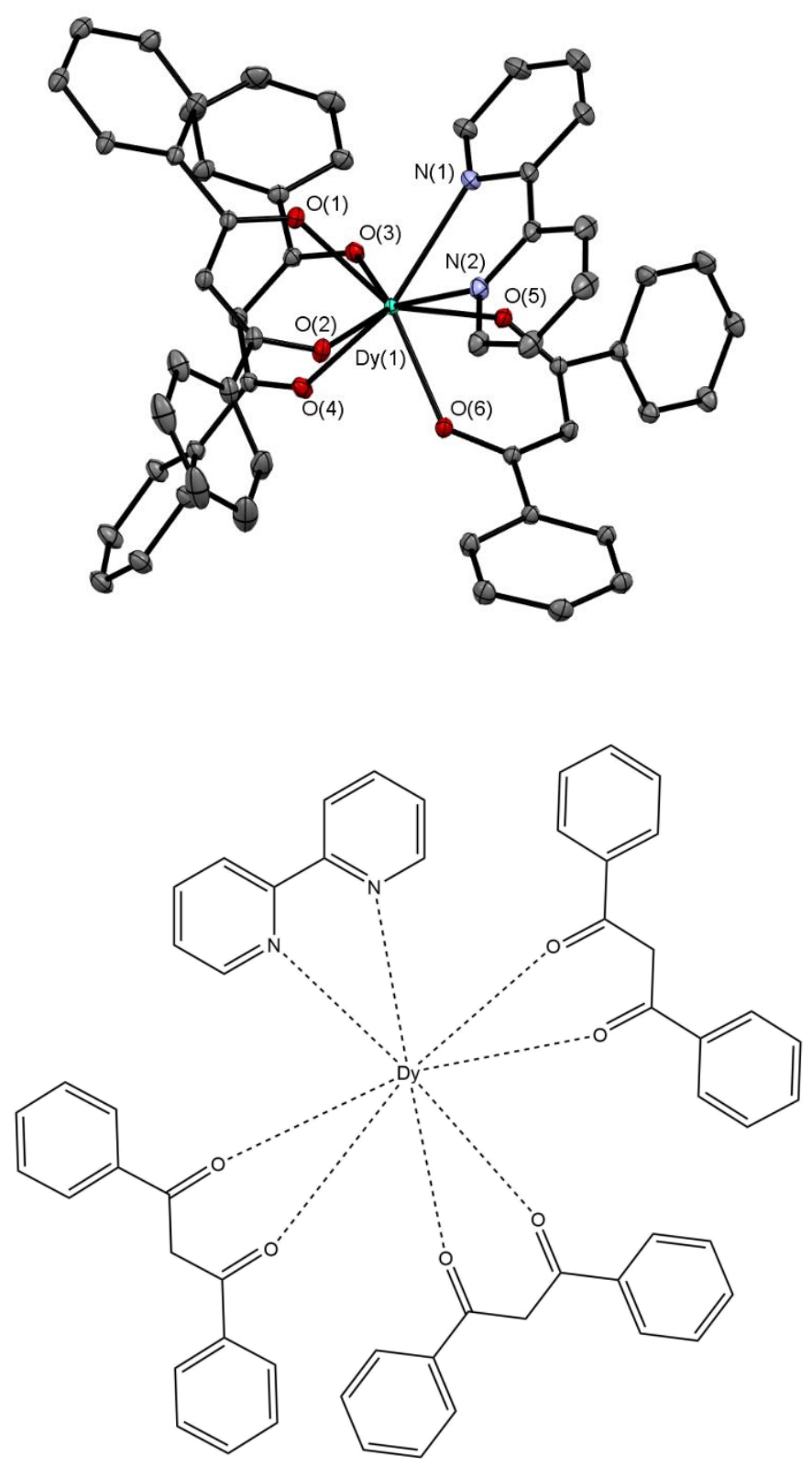
a)

C)

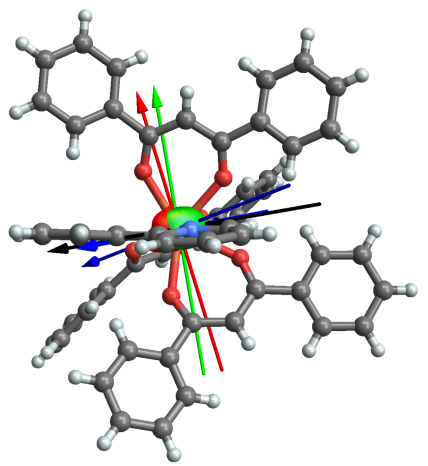

b)

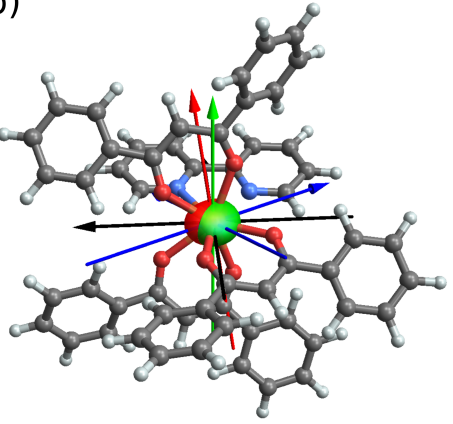

d)

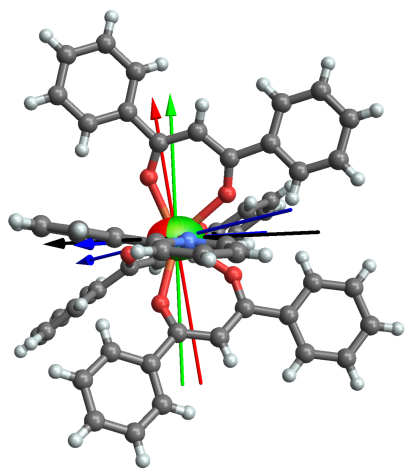




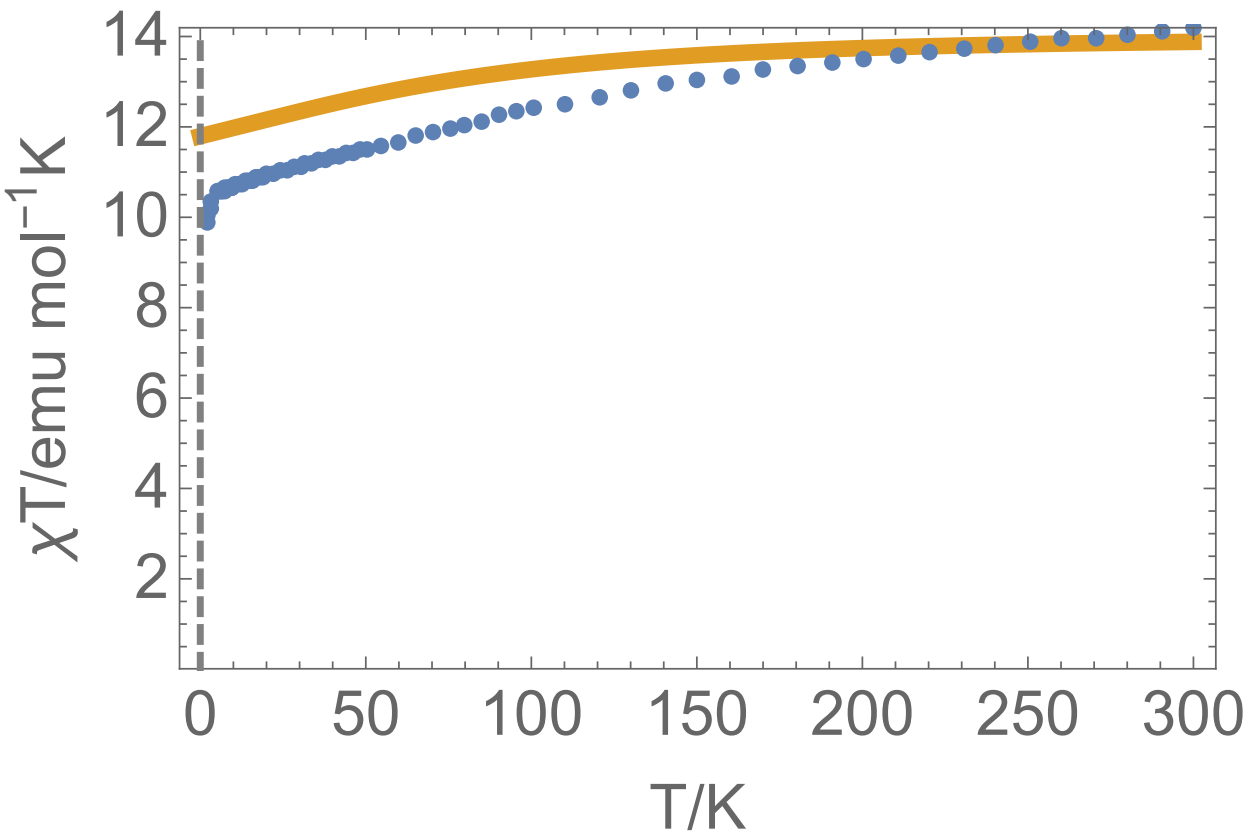



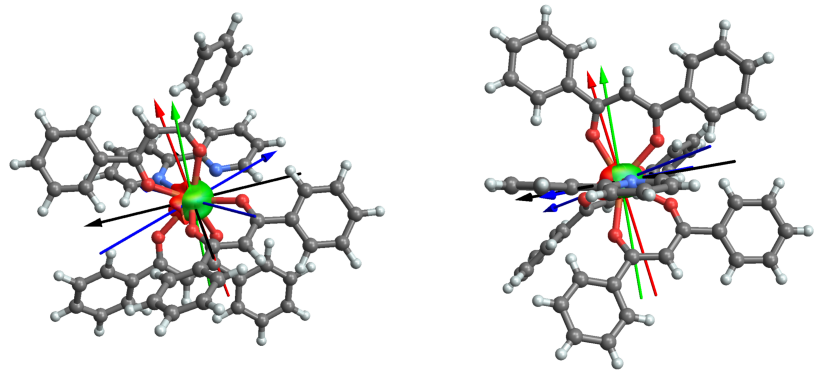